\title{
Three-Dimensional High-Resolution Temporal Bone Histopathology Identifies Areas of Vascular Vulnerability in the Inner Ear
}

\author{
Bela Bükia,b Antonia Mair ${ }^{a, b} \quad$ Jacob M. Pogson ${ }^{c, d} \quad$ Nicholas S. Andresen ${ }^{e}$ \\ Bryan K. Ward ${ }^{\mathrm{e}}$ \\ ${ }^{a}$ Karl Landsteiner University of Health Sciences, Krems, Austria; ${ }^{b}$ Department of Otorhinolaryngology, University \\ Hospital Krems, Krems, Austria; 'Neurology Department, School of Medicine, The Johns Hopkins University, \\ Baltimore, MD, USA; ${ }^{d}$ Neurology Department, Royal Prince Alfred Hospital, Camperdown, NSW, Australia; \\ eDepartment of Otolaryngology-Head and Neck Surgery, The Johns Hopkins University School of Medicine, \\ Baltimore, MD, USA
}

\section{Keywords}

Inner ear · Labyrinth · Vestibular neuritis · Vascular disease ·

Blood supply · Circulation

\begin{abstract}
Objectives: Hypothesized causes of vestibular neuritis/labyrinthitis include neuroinflammatory or vascular disorders, yet vascular disorders of the inner ear are poorly understood. Guided by known microvascular diseases of the retina, we developed 2 hypotheses: (1) there exist vascular vulnerabilities of artery channels in cases of hypothetical nerve swelling for the superior, inferior, and vestibulocochlear artery and (2) there are arteriovenous crossings that could compromise vascular flow in disease states. Methods: Two fully mounted and stained temporal bones were used to render three-dimensional reconstructions of the labyrinth blood supply. Using these maps, areas of potential vascular compression were quantified in 50 human temporal bones. Results: Although inner ear arteries and veins mostly travel within their own bony channels, they may be exposed (1) at the entrance into the otic capsule, and (2) where the superior vestibular vein crosses the inferior vestibular artery. At the entry into the otic capsule, the ratio of the soft tissue to total space for
\end{abstract}

karger@karger.com www.karger.com/aud

Karger $\stackrel{\text { ' }}{5}$
(C) 2021 The Author(s)

Published by S. Karger AG, Basel

This is an Open Access article licensed under the Creative Commons Attribution-NonCommercial-4.0 International License (CC BY-NC) (http://www.karger.com/Services/OpenAccessLicense), applicable to the online version of the article only. Usage and distribution for commercial purposes requires written permission. the superior vestibular artery was significantly greater than the inferior vestibular artery/cochleovestibular artery (median 44, interquartile range 34-55 vs. 14 [9-17], $p<0.0001$ ). Conclusions: Three-dimensional reconstruction of human temporal bone histopathology can guide vascular studies of the human inner ear. Studies of retinal microvascular disease helped identify areas of vascular vulnerability in cases of hypothetical nerve swelling at the entrance into the otic capsule and at an arteriovenous crossing near the saccular macula. These data may help explain patterns of clinical findings in peripheral vestibular lesions.

(C) 2021 The Author(s).

Published by S. Karger AG, Basel

\section{Introduction}

Vestibular neuritis or neuronitis (VN) is a clinical syndrome of spontaneous prolonged vertigo from sudden unilateral disruption of the peripheral vestibular system [Baloh, 2003]. When hearing loss is also present, the clinical syndrome has been called labyrinthitis [Goddard and Fayad, 2011]. Although the etiology of VN remains uncertain, a hypothesized cause is reactivation of latent herpes simplex virus, similar to the proposed cause of acute
Correspondence to:

Bela Büki, bela.bueki@krems.lknoe.at 
idiopathic facial nerve paresis known as Bell's palsy [Nadol, 1995; Jeong et al., 2013; Rujescu et al., 2018]. Nerves become edematous in response to injury [Mizisin et al., 1990]. In patients with Bell's palsy, for example, surgeons have observed swelling of the facial nerve [Yanagihara et al., 2000]. Similar edema of the vestibular nerves within narrow bony channels has been hypothesized to disrupt neural transmission and cause VN [Goebel et al., 2001; Gianoli et al., 2005; Buki and Ward, 2021].

Traditionally, the diagnosis of $\mathrm{VN}$ was supported by asymmetrically reduced responses to a caloric stimulus. The caloric test assesses only the lateral semicircular canal and uses a non-physiological, low-frequency stimulus. Recent studies have incorporated other clinical tests such as the video head impulse test, pure tone audiometry, and vestibular-evoked myogenic potentials to assess all sensory organs within the inner ear. The findings from these studies have revealed complex patterns of end organ impairment in VN [Pogson et al., 2016; Taylor et al., 2016]. For instance, several studies found a correlation between impaired vestibulo-ocular reflex testing of the posterior semicircular canal and hearing loss [Aw et al., 2001; Rambold et al., 2005; Pogson et al., 2016; Tarnutzer et al., 2017]. The more complicated patterns suggest clinical symptoms of $\mathrm{VN}$ and labyrinthitis may reflect a common presentation of several disease processes. Additional hypotheses, such as disrupted blood supply, have been proposed by some authors to explain these patterns of peripheral loss in labyrinthitis [Rambold et al., 2005; Pogson et al., 2016].

The blood supply of the inner ear sensory epithelia is the labyrinthine artery, an end artery that branches within the internal auditory meatus into the superior vestibular artery and common cochlear artery. On its way to supplying the saccule and posterior semicircular canal, the common cochlear artery then becomes the vestibulocochlear artery and then the inferior vestibular artery, after providing the basal cochlear artery branch to supply the basal turn of the cochlea. Four veins drain the labyrinth [Mazzoni, 1990; Guo et al., 2016]. The superior and inferior vestibular veins merge with a third, the cochlear vein, to form the cochleovestibular vein. The cochleovestibular vein runs inferior to the cochlea in a separate bony channel near the cochlear aqueduct to reach the inferior petrosal sinus. The fourth vein, the posterior vestibular vein, is independent of the other 3 and also drains the semicircular canals and posterior utricle, running parallel to the endolymphatic duct in a separate bony channel before reaching the inferior petrosal sinus or the jugular bulb [Mazzoni, 1979].
Vascular diseases of the inner ear in humans are poorly understood; however, analogies may be drawn from other organs. Like the labyrinth, the eye contains a sensory epithelium supplied by a single end artery; however, the human retina has numerous well-characterized vascular disorders associated with relatively well-described sensory impairment. External pressure applied to retinal microvasculature, for instance, can lead to sensory impairment and may have corollary pathologies in the inner ear. Edema of the optic nerve causes secondary effects on the retinal vasculature, blocking blood flow, and exacerbating vision impairment [Joussen, 2007; Hayreh, 2016]. Stiffening of retinal arteries from chronic hypertension can occlude adjacent retinal veins at arteriovenous crossings, resulting in vision loss [Duker and Brown, 1989; Scott et al., 2020]. Within the ear, edematous vestibular nerves might displace or compress accompanying vessels, thereby causing secondary disturbances of blood flow, as has been suggested by [Goebel et al., 2001]. In addition, any location where arteries cross veins in the inner ear can increase the risk of secondary venous occlusion.

Our aim in this study was to map the arteries and veins of the human inner ear in 2 fully mounted and stained temporal bones and to render 3-dimensional reconstructions of the labyrinth blood supply to test hypotheses based on comparable diseases in the retina. We hypothesized that there would be areas in which arteries supplying the vestibular sensory epithelia would be at increased risk of compression by edema of adjacent vestibular nerves. This hypothesis was tested by quantifying areas of potential vascular compression in 50 human temporal bones. Using the 2 fully mounted temporal bones, we secondarily hypothesized that locations where arteries and veins cross could be identified as areas at risk of venous occlusion.

\section{Materials and Methods}

\section{Mapping the Blood Supply in 2 Fully Mounted and Stained} Ears

Two fully mounted normal ears from the temporal bone collection of the Otopathology Laboratory at the Massachusetts Eye and Ear Infirmary were examined (NIDCD National Temporal Bone Registry; IRB ethical commission permission number: 9204-017X). The donor of the right ear was a 2-day-old female infant (unknown postmortem time), the donor of the left ear was a 71-year-old male (postmortem time $14 \mathrm{~h}$ ). The 71-year-old donor had no known previous cochleovestibular complaints. These 2 ears were sectioned with a slice thickness of $20 \mu \mathrm{m}$ and stained with hematoxylin and eosin. Each section was mounted. The histology slides were scanned using Aperio Image Scan Version 12.4.0.5043 and images of the scans were manually aligned as $8,000 \times 5,000$ 


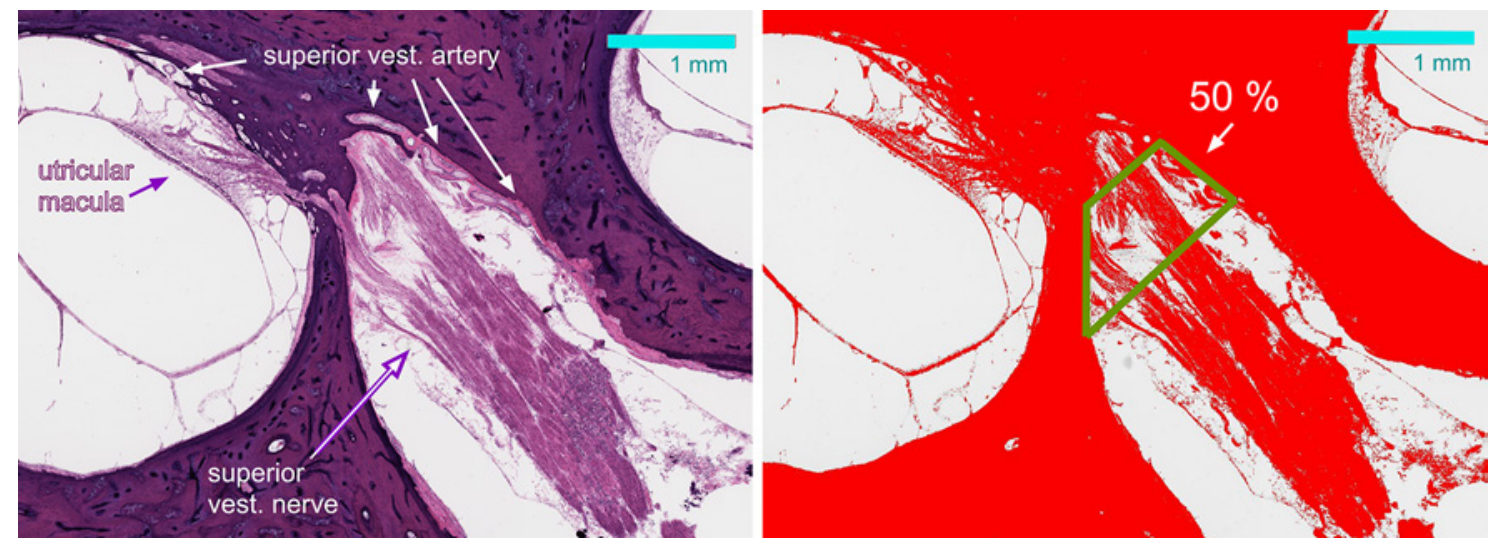

Fig. 1. Demonstration of the area measurement method using Fiji ImageJ 2.0.0.

pixel JPEG files using FIJI ImageJ 2.0.0 (National Institutes of Health, Bethesda, MD, USA; https://imagej.nih.gov/ij/download. $\mathrm{html}$ ). Blood vessels and inner ear sensory epithelia were manually registered using 3D Slicer as 3,000 × 2,000 pixel JPEG files (Slicer version 4.10.2 http://www.slicer.org) [Fedorov et al., 2012]. Using the Aperio scans and high-resolution JPEGs, it was possible to identify and register vessels (arteries and veins) with a diameter of at least $15 \mu \mathrm{m}$. The STL files created by Slicer were imported into Blender for display (Blender v.2.91.2, Stichting Blender Foundation, Amsterdam, The Netherlands, http://www.blender.org). Guided by pathophysiology of retinal microvasculature, reconstructed arteries and veins from these specimens were reviewed for vulnerabilities.

Statistical Analysis of Regions of Interest in 50 Normal Ears

Fifty normal ears from the temporal bone collection of the Otopathology Laboratory at the Massachusetts Eye and Ear Infirmary were then examined. The samples used in this study had been collected by the NIDCD National Temporal Bone Registry, Otopathology Laboratory at Massachusetts Eye and Ear Infirmary, Boston. The study was carried out according to the procedures approved by the Institutional Review Board (NIDCD National Temporal Bone Registry; IRB ethical commission permission number: 92-04-017X), and donors had signed the written "National Temporal Bone Donor Program Donor Consent Form" during the enrollment procedure. Cases were chosen if their clinical data indicated no previous vestibular symptoms. Twenty-five left ears and 25 right ears were analyzed from 21 female and 29 male donors. Average age at death was 56 years (range: 2 days- 87 years). Specimens were harvested on average $13 \mathrm{~h}$ postmortem. All ears were sectioned horizontally (i.e., in the axial plane) with a slice thickness of $20 \mu \mathrm{m}$. Every 10th preparation was stained and mounted. The regions of interest in the preparations were captured using CAPTAVISION V 3.9 on Olympus Microscope BX51 (Objective $1.25 \times$ ) + Excelis Camera by Accu-Scope with a capture resolution: $3,264 \times 1,836$ and stored in high-quality JPEG format.

The images were analyzed using FIJI ImageJ 2.0.0 software. All stained sections of all ears were examined carefully and the locations where superior vestibular artery and the vestibulocochlear or inferior vestibular arteries perforated the bony capsule of the inner ear were identified. Then, using the area measurement tool of the
FIJI ImageJ software, we measured the area of the nerve occupying the bony channel (shown in Fig. 1). Image analysis was performed independently by 2 examiners after a joint training session.

The artery was classified as "vulnerable" if the surrounding soft tissue (including the artery itself) filled more than two-thirds $(66 \%)$ of the bony channel in the region of interest, that is, where the artery enters its bony channel. Artifacts during tissue preparation that produce the "normal" histologic appearance include $10 \%$ shrinkage of most tissues due to fixation [Schuknecht et al., 2010].

After independent analysis, the inter-rater reliability was calculated. This value was $K=1.000$ (Cohen's kappa) for classifying the artery as "vulnerable" or "non-vulnerable" based on the percentage of nerve in the channel. Cohen's kappa was 0.924 for the mean area of the nerve in the channel for the superior vestibular artery and 0.919 for mean area of the nerve in the channel for the vestibulocochlear/inferior vestibular artery. Test of normality was performed using the D'Agostino \& Pearson omnibus normality test. Between-group comparisons were performed using the MannWhitney $U$ test with a $p$ value $\leq 0.05$ for statistical significance. Statistical analyses were performed using SPSS (version 26.0, IBM, Armonk, NY, USA).

\section{Results}

\section{Three-Dimensional Mapping of the Cochlear Blood}

\section{Supply in 2 Fully Mounted Specimens}

Figure 2 shows the reconstruction of the 2 fully mounted and stained ears and Figure 3 shows the map of the arteries and veins. After leaving the internal auditory meatus, during most of their course through otic capsule bone, the arteries travel in their own bony channels. From the edge of the specimen starting in the distal internal auditory canal, the arteries from both donors share a similar morphology. The superior vestibular artery branches first from the labyrinthine artery and supplies the utricle, lateral, and superior semicircular canal sensory epithelia. 
Fig. 2. Three-dimensional reconstruction of the 2 fully mounted and stained ears from the 71-year-old (a) and 2-day-old (b) donors. Yellow: sensory epithelium; green: basilar membrane; red: arteries; blue: veins. Upper panels: view from inferior (left ear, a; right ear, b), lower panels: view from lateral. For explanation of anatomical structures, see Fig. 3.

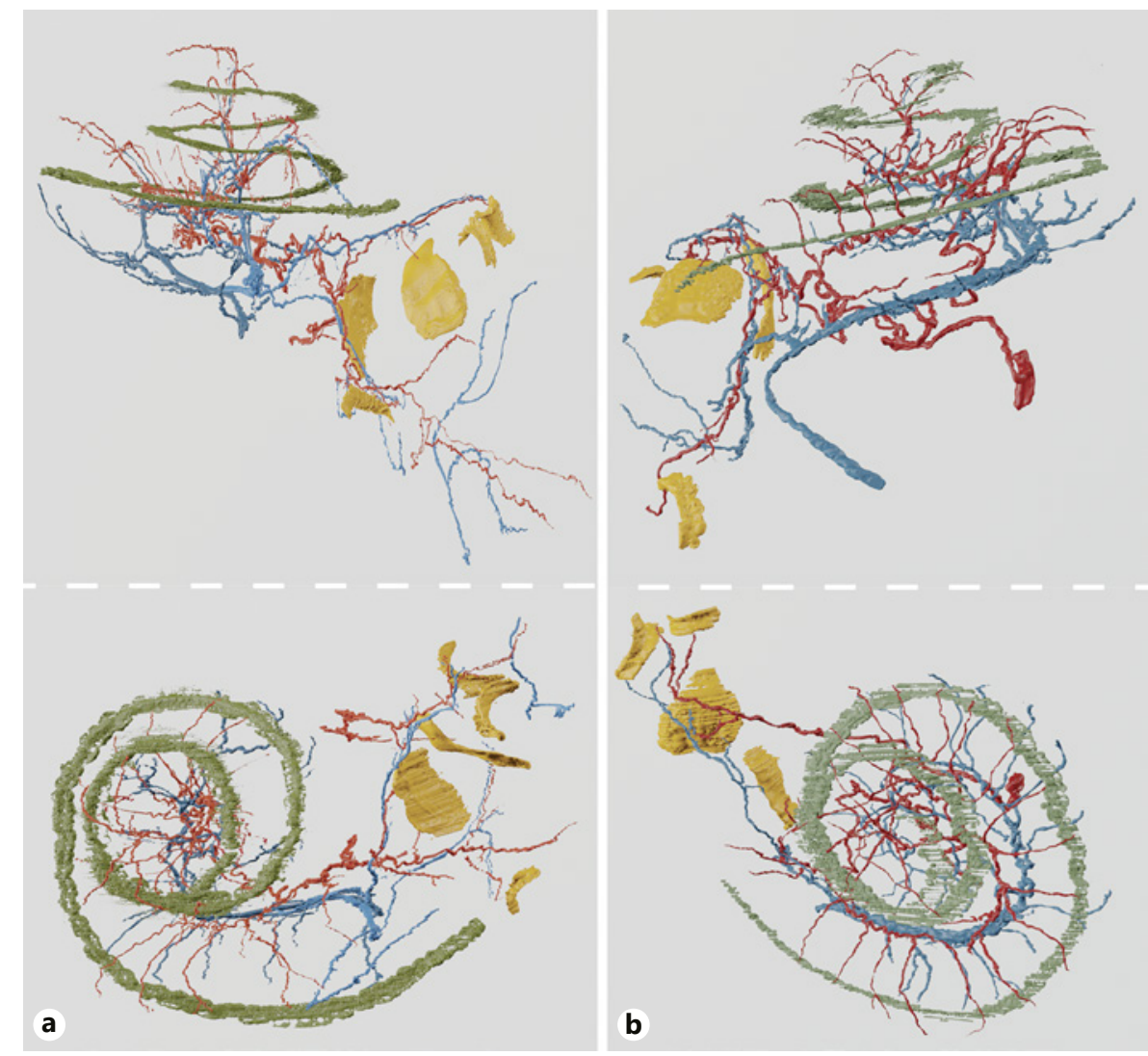

The common cochlear artery bifurcates into the cochlear artery and the vestibulocochlear artery. One branch of the vestibulocochlear artery, the basal cochlear artery, supplies the basal cochlea, and the other branch, the inferior vestibular artery, supplies the saccule and the posterior semicircular canal crista. Care was taken to look for arterioles connecting the tip region of the cochlear artery and the basal cochlear artery, but an anastomosis was not found in either ear. Also, an arteriole was sought arising from the superior vestibular artery and supplying the upper part of the saccular macula but was not found in either ear. In the 2-day-old donor, the vestibulocochlear artery divides into the basal cochlear and inferior vestibular arteries prior to entering the otic capsule bone, whereas in the 71-year-old donor, the vestibulocochlear artery divides after entering the otic capsule bone.

The veins traveled in their own bony channels. In both specimens, the superior and inferior vestibular veins joined the cochlear vein to form the cochleovestibular vein that courses near the cochlear aqueduct. In the 71-year-old donor, a secondary venous pathway was identified draining the labyrinth sensory epithelia into the posterior vestibular vein that runs near, but in a sepa- rate bony channel from, the vestibular aqueduct. The diameter of this venous drainage pathway in the 2-year-old infant was too small to register accurately.

We examined areas where the labyrinth vessels were exposed to adjacent structures that could be affected by pathology, secondarily compromising the vasculature. The arteries appeared most vulnerable at the transition from the internal auditory meatus into the bony labyrinth (Fig. 4). We also found in both ears 1 area of arteriovenous crossing; in both ears, the inferior vestibular artery runs near and then crosses the superior vestibular vein (Fig. 5).

\section{Identifying Vulnerable Regions of the Arterial System} in 50 Normal Ears

Photographs from the larger cohort were at a lower spatial resolution than the fully mounted specimens. Every tenth section ( $20 \mu \mathrm{m}$ thickness) was mounted such that there was a $0.18 \mathrm{~mm}$ gap between sections. Nevertheless, examination of the terminal branches of the superior and inferior vestibular arteries led to the following observations. 
Fig. 3. Labeled cartoon from a flattened 3-dimensional reconstruction of the arteries (a) and veins (b) of a fully mounted and stained ear. The upper part of the cochlear artery has been removed in (a). The lower image (b) includes the venous drainage into the posterior vestibular vein near the vestibular aqueduct. This structure could be traced only in the specimen from the 71-year-old donor as the veins were too small for accurate registration in the specimen from the 2-day-old donor. SCC, semicircular canal; AICA, anterior inferior cerebellar artery.

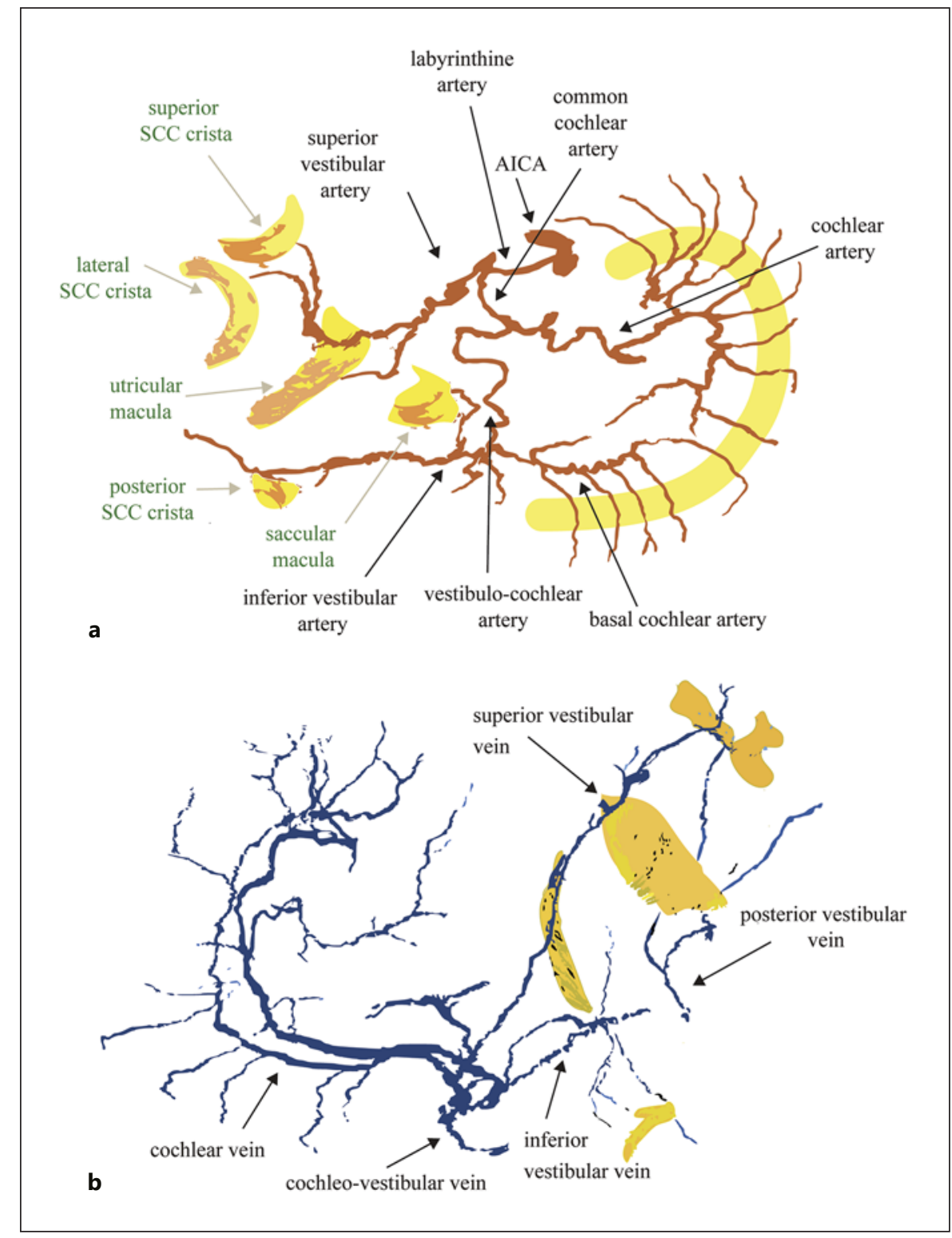

In the medial to lateral direction, without exception, the superior vestibular artery entered the temporal bone in the anterosuperior part of the fundus of the internal auditory meatus and traveled in a separate bony channel parallel to the branches of the auditory and vestibular nerves. After dividing from the labyrinthine artery, the vestibulocochlear or inferior vestibular artery also traveled in its own bony channel, likewise separate and parallel to the inferior vestibular nerve. The entry region of the vestibulocochlear or inferior vestibular artery into the bone was at a relatively wide part of the internal auditory meatus. Figure 5 shows examples of the anatomy of the superior vestibular artery and the vestibulocochlear artery. The vestibulocochlear artery branched into the basal cochlear artery and the inferior vestibular artery. The inferior vestibular artery then divided into the saccular artery and an artery that supplied the crista of the posterior semicircular canal. The artery that supplied the saccule initially followed an anterosuperior direction and arched caudally to reach the saccule from a superior position. The artery of the posterior semicircular canal crista approached the singular nerve channel containing the nerve to the posterior semicircular canal only at the most lateral end, immediately prior to entering the crista. In 1 

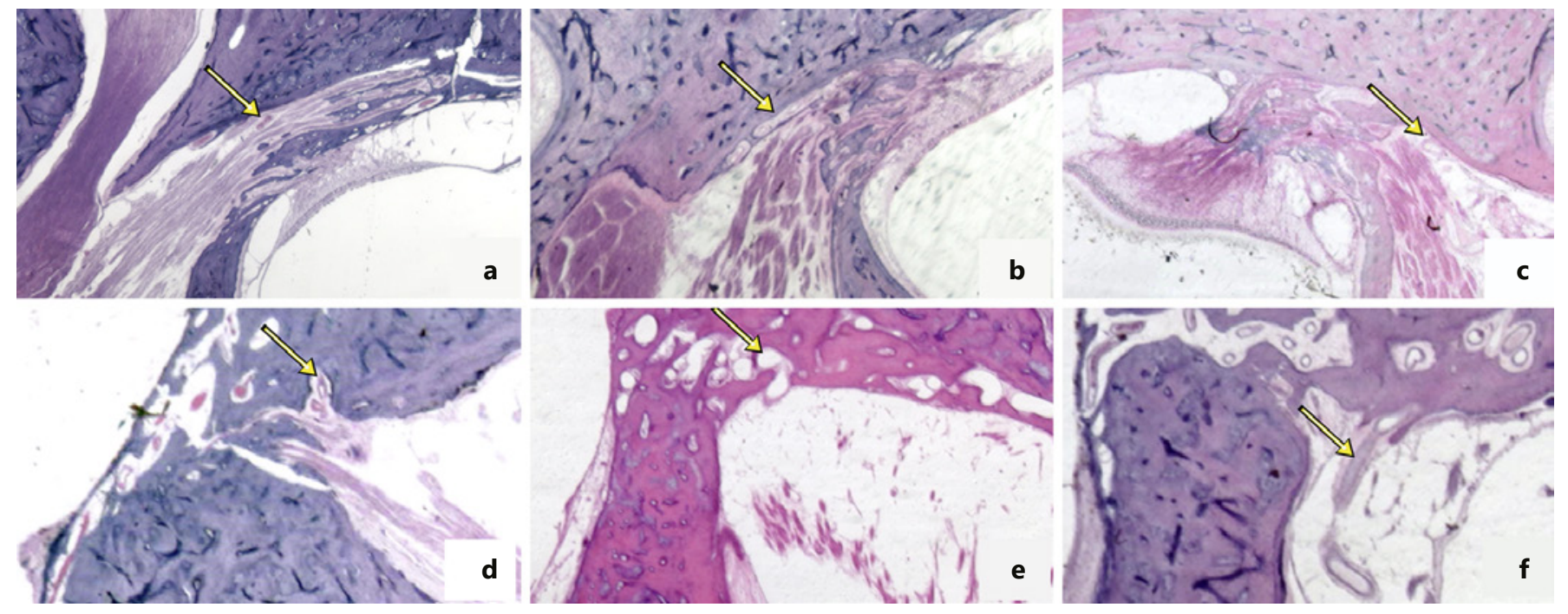

Fig. 4. Anatomic examples from the regions of interest for the superior vestibular artery (upper row, a-c) and vestibulocochlear/inferior vestibular artery (lower row, d-f). Arrows denote arteries. Dense soft tissue in the bony channel (a); artery is shielded by its own bony channel (b); artery is exposed (c); artery runs in a densely packed bony channel (only in this ear was the inferior vestibular artery considered to be vulnerable to compression) (d); there are no nerve fibers around the region (e-f), where the vestibulocochlear artery/inferior vestibular artery enters the bony channel.

ear (No. 77), a tiny saccular artery variant was found arising directly from the common cochlear artery (as previously reported in [Mazzoni, 1990]).

The ratio of the soft tissue to the total space was measured at the entry into the otic capsule bone to assess the vulnerability of the artery to compression by an adjacent, hypothetically edematous vestibular nerve. In the case of the superior vestibular nerve $(n=46)$, the soft tissue filled the canal in average $( \pm$ SD) by $46 \pm 17 \%$ (median: 44 , min: $15,25 \%$ percentile: $34,75 \%$ percentile 55 , max: 95 ), in the case of the inferior nerve $(n=44)$ by $17 \pm 14 \%$ (median: 14 , min: $0,25 \%$ percentile: $9,75 \%$ percentile: 17 , max: 68 , two-tailed Mann-Whitney U test, $p<0.0001$, Fig. 6).

In 4 ears, the region immediately before the superior vestibular artery entered its bony channel could not be identified because the location was not stained. In 8 of the 46 ears (17\%), the nerve fibers were denser than $66 \%$ of the cross-sectional area in the region where the superior vestibular artery enters its bony channel (Fig. 6) and were $>80 \%$ in 2 of these ears (4\%). In 6 ears, the region of interest concerning the inferior vestibular or vestibulocochlear artery could not be identified in a stained section. In 1 ear $(2 \%)$, the inferior vestibular or vestibulocochlear artery entered its bony channel where the soft tissues were dense ( $68 \%$ of the cross-sectional area).

\section{Discussion}

In summary, in most cases ( 36 of $46,78 \%$ ), the superior vestibular artery enters a separate bony channel from the superior vestibular nerve in a region with a density of nerve fibers less than two-thirds the cross-sectional area. In 8 of 46 ears (17\%), the nerve fiber density exceeded two-thirds the cross-sectional area, and in 2 of 46 (4\%) ears, density was more than $80 \%$. Evidence from nerve injury studies indicate edema is a common sequela and can be more than triple the cross-sectional area of the affected nerve [Weiss, 1943]. By comparison, the inferior vestibular or vestibulocochlear artery appeared less vulnerable to edema from the nearby inferior vestibular nerve fibers because of the lower density of nerve fibers near the accompanying artery.

According to our data, the anatomy of the labyrinth could make the superior vestibular artery more vulnerable to external compression if the adjacent superior vestibular nerve becomes edematous in response to injury or inflammation or is affected by tumor. We are unable to find similar investigations in the literature. In the retina, edema of the optic nerve is believed to secondarily affect thin-walled veins and capillaries [Hayreh, 2016]. Arteries have thicker walls to withstand higher intraluminal pressures. Nevertheless, clinical syndromes caused by exter- 


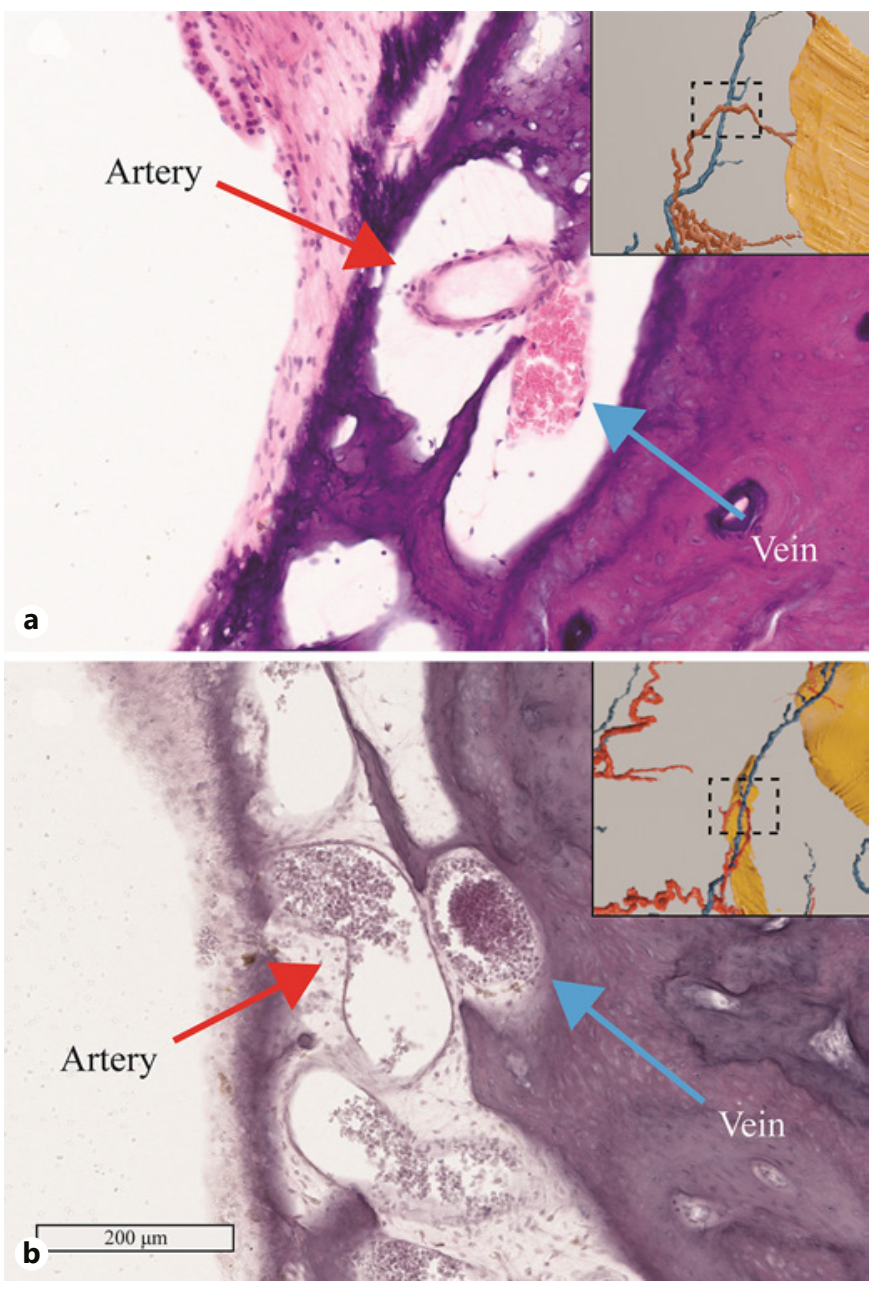

Fig. 5. a, b In each of the 2 fully histologically processed ears, we found 1 region of arteriovenous crossing. In both ears, the superior vestibular vein and the inferior vestibular artery run together in the region of the saccule. Areas of arteriovenous crossings can be sites for pathology in several retinal microvascular diseases.

nal compression of an artery have been reported for several larger arteries such as the subclavian artery [Hussain et al., 2016], left main coronary artery [Akbal et al., 2018], and celiac artery [Horton et al., 2005], among others. In each example, however, these syndromes are rare causes of patient symptoms. We hypothesize that secondary causes of external compression of the vestibular arteries is uncommon but could affect more frequently the superior vestibular artery and the structures it supplies.

External compression of veins appears to be more common in medicine than compression of arteries, likely due to veins having thinner walls and lower intraluminal pressure. This is supported by models of compartment syndrome in which veins become occluded prior to arteries contained within a swelling fascia compartment [Shrier and Magder, 1995]. In the inner ear, the primary venous drainage is via 2 veins: the cochleovestibular vein near the cochlear aqueduct and the posterior vestibular vein near the vestibular aqueduct. In both, the veins traverse a bony channel, presumably providing protection from external compression. While the veins do not appear to be vulnerable to adjacent neural edema, an arteriovenous crossing was found in both high-resolution ears where the inferior vestibular artery crosses the superior vestibular vein. To our knowledge, vascular disease here in the ear has not been reported, but this location should be monitored for disease. Sites of arteriovenous crossings are known risk factors for venous occlusion in the retina and in diseases like Takayasu's arteritis in which retinal arteriovenous shunts develop [Tanaka and Shimizu, 1987; Staurenghi et al., 1994].

Similar to external pressure on arterioles, we hypothesize that veins are vulnerable where they exit the bone and drain into the venous sinuses. Studies from guinea pigs in which the venous drainage of the inner ear is occluded indicate profound loss of inner ear function [Kimura and Perlman, 1956a, b; Kimura et al., 1996]. Human cases of hearing loss and vertigo have been reported for venous sinus occlusion in the absence of ear disease [Fonseca et al., 2008; Kim et al., 2008; Gattringer et al., 2012]. The effects of venous occlusion in human inner ear disease are worth additional study. In current practice, injury to the cochleovestibular vein during infracochlear approaches to the petrous apex or when packing the inferior petrosal sinus during surgery for glomus jugulare could explain cases of postoperative sensorineural hearing loss. Similarly, occlusion of the posterior vestibular vein could explain vestibular symptoms after procedures on the endolymphatic sac.

Recent studies of VN and labyrinthitis have considered intraluminal arterial occlusion in the pathophysiology [Taylor et al., 2016]. Intraluminal arterial obstruction causes a large proportion of retinal disease [Sheyman and Fawzi, 2020] and likely contributes similarly to pathology within the labyrinth. In the eye, central retinal artery occlusion may result from embolic arteriole blockage, causing retinal infarction and vision loss [Scott et al., 2020]. Similarly, ocular ischemic syndrome may occur in patients with atherosclerosis and result in damage to the retina through chronic inadequate blood supply [Sheyman and Fawzi, 2020].

In Figure 7, we show an analysis of data from Taylor et al. [2016] with consideration to intraluminal vascular oc- 
Fig. 6. Statistical results of area measurements. a Column statistics (mean $\pm \mathrm{SD}$ ). b Histogram.
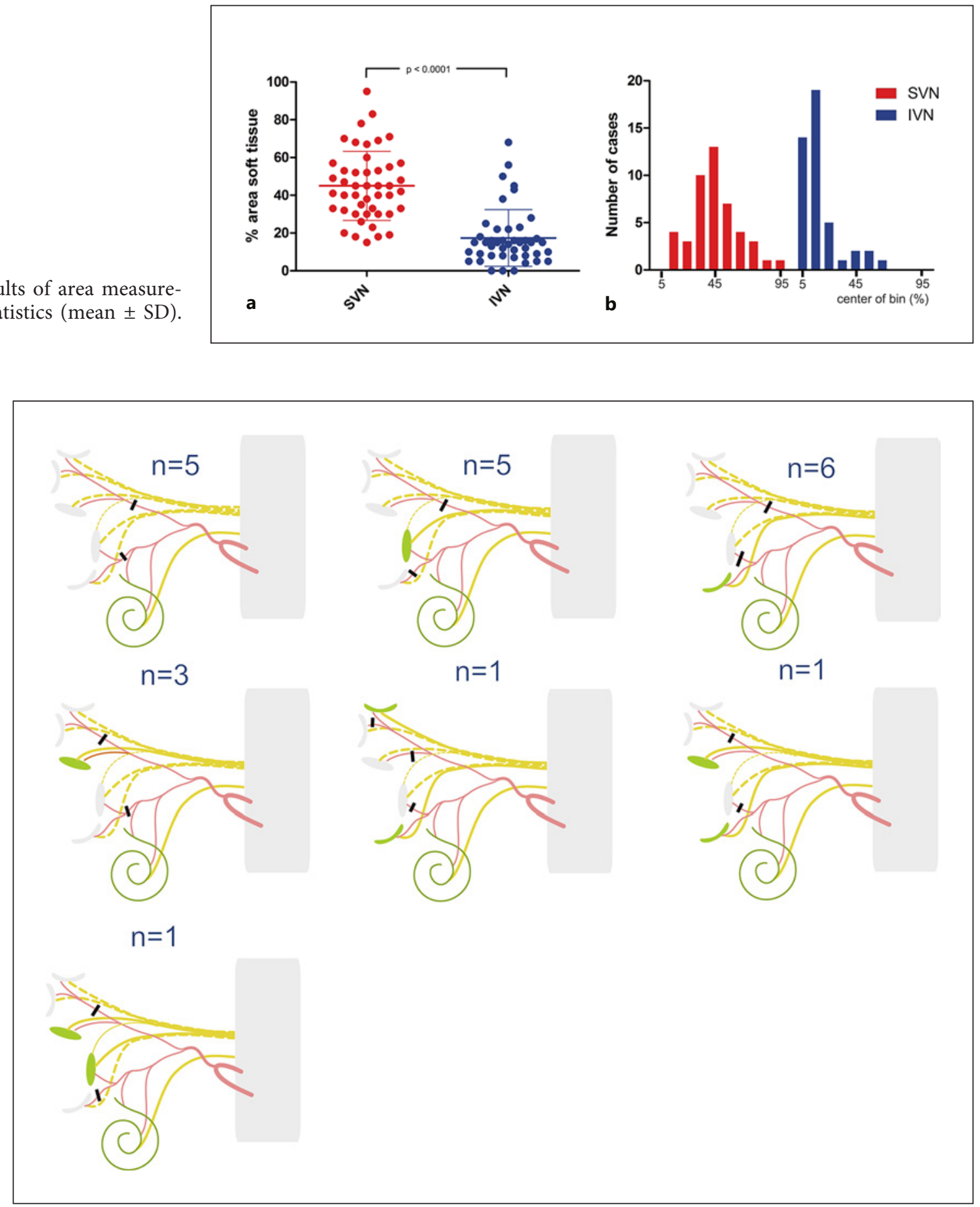

Fig. 7. Hypothetical locations of arterial blockage calculated from the data of Taylor et al. [2016]. Taylor et al. [2016] examined all 5 vestibular end organs in 43 patients after acute VN. In the majority of cases, the patchy distribution of the lesions does not make it possible to explain the pathology by a single hypothetical vascular cause. Dashed yellow lines: involved nerves; yellow lines: intact nerves; red line: arteries; green lines: functional, intact sensory end organs (legend see also in Fig. 3). Twenty-two cases are shown from their data, in which both vestibular nerves were involved. Short black lines highlight where arterial obstruction could lead to the particular pattern of dysfunction in the individual groups. As these lines show, hypothetical acute blockage of blood supply would not be a simple explanation for the acute vestibular symptoms since in all cases shown at least 2 simultaneous locations should be assumed. 
clusion [Taylor et al., 2016]. Although several cases could be explained by a vascular distribution, there are several others where an explanation based on arterial vascular supply is inadequate. To date, archival temporal bone studies have provided little evidence of intraluminal blood flow obstruction secondary to atherosclerotic plaques within the ear. However, archival temporal bones are decalcified for weeks to months in ethylene diamine tetra-acetic acid [Schuknecht, 1993], which is known to remove atherosclerotic plaques and has been used as a treatment for atherosclerosis [Song and Zhang, 2020]. Consequently, it is likely that archival temporal bone studies underestimate the role of intraluminal blood flow obstruction secondary to atherosclerosis. Additionally, in disorders such as sickle cell disease and Susac syndrome, microvascular arterial disruption occurs through the "sickling" of red blood cells and endothelial cell disruption, respectively. Given that atherosclerosis [Kim et al., 2020], sickle cell disease [Strum et al., 2021], and Susac syndrome [Kleffner et al., 2016] are associated with hearing loss and vascular eye disease, it is likely that similar vascular processes occur in the ear and eye.

The study of vascular diseases of the human inner ear remains in its infancy due to the poor spatial and temporal resolution of current imaging technology. Even conventional histology methods that involve decalcification and staining every 10th section make it difficult to follow the small vessels of the inner ear. Previous studies that mapped the labyrinthine blood supply were performed with arterial injection of colored medium that was then visualized with stereo-microscope photography [Mazzoni, 1990] and more recently with 3-dimensional microcomputed tomography and synchrotron radiation phase contrast imaging [Iyer et al., 2018; Mei et al., 2018]. We reconstructed a 3 -dimensional model of the labyrinth arterial and venous blood supply to $20 \mu \mathrm{m}$ slices using manual segmentation of a fully stained and mounted temporal bone. This process currently is labor intensive but has an advantage in resolution, keeping the bony anatomy intact, and in correlations with end organ pathology. In the 2 fully mounted specimens, the known blood vessel branches could be identified. In contrast to current labyrinth vascular depictions [Baloh and Kerber, 2011], however, 2 significant arteriole branches were not found: (1) an anastomoses between the tip of the cochlear artery from the basal cochlear artery, and (2) a branch from the superior vestibular artery to the superior saccular macula. These missing connections may be significant when considering cases of arterial occlusion, for example, whether the saccular macula is only supplied by the inferior ves- tibular artery or also by the superior vestibular artery - in a pattern similar to the nerves. According to [Mazzoni, 1990; Nabeya, 1923], the saccular macula is only supplied by a branch of the inferior vestibular artery.

Our approach here was to provide a framework whereby future studies could explore vascular disease of the inner ear. In ophthalmology, the microvasculature of the retina can be seen by fundus photography, intravenous contrast studies, and optical coherence tomography. Technology development is needed to achieve a similar level of spatial and temporal resolution in the inner ear; nevertheless, we hypothesize that microvascular diseases of the retina may serve as a useful guide as we improve our understanding of inner ear vascular disorders.

\section{Conclusion}

Three-dimensional reconstructions of human temporal bone histopathology can guide vascular studies of the inner ear. Inner ear arteries and veins travel within their own bony channels through much of their course. We found that the superior vestibular artery appears more vulnerable to external compression than the inferior vestibular and vestibulocochlear arteries.

\section{Acknowledgments}

The authors thank Joseph B. Nadol, Jr. and MengYu Zhu from the NIDCD National Temporal Bone, Hearing \& Balance Pathology Resource Registry for granting access of the histological preparations and for their support in 2020. B.B. and A.M. appreciate the contribution of NÖ Landesgesundheitsagentur, legal entity of University Hospitals in Lower Austria, for providing the organizational framework to conduct this research and for financial support through its Open Access Funding Programme.

\section{Statement of Ethics}

The study protocol was in accordance with the ethical standards established in the 1964/2013 (7th revision) Declaration of Helsinki for research involving human subjects. The samples used in this study had been collected by the NIDCD National Temporal Bone Registry, Otopathology Laboratory at Massachusetts Eye and Ear Infirmary, Boston. The study was carried out according to the procedures approved by the Institutional Review Board (NIDCD National Temporal Bone Registry; IRB ethical commission permission number: 92-04-017X), and donors had signed the written "National Temporal Bone Donor Program Donor Consent Form" during the enrollment procedure. 


\section{Conflict of Interest Statement}

B.W. is supported by grants from the NIH (K23DC018302) and the Hearing Health Foundation to study the effects of magnetic vestibular stimulation and to improve MRI in healthy adults and patients with Meniere's disease and has a patent US10646723 that is unrelated to the current work.M.A., J.P., and N.A.have no conflicts of interest to declare. B.B. receives royalties from Oxford University Press (see also Funding Sources below).

\section{Funding Sources}

B.B. was supported by the Grants RTO005 and SF06 of the Karl Landsteiner Private University of Health Sciences, Austria. N.A. was supported by Grant 2T32DC000027-31A1 from the National Institute of Health (NIDCD). J.P. is supported by a Junior Fellowship from the Garnett Passe and Rodney Williams Memorial Foundation and by philanthropy received from the Cinquegrana, Lott, and Schwerin families through Prof. David Zee at Johns Hop- kins University. B.W. was supported by clinician-scientist awards from Johns Hopkins University and the American Otological Society, as well as K23DC018302.

\section{Author Contributions}

B.B., B.W., N.A., and J.P. contributed to the study design. B.B. performed the $3 \mathrm{D}$ reconstructions of the fully mounted specimens that were then reviewed by B.B. and B.W. B.B. and A.M. quantified the 50 normal temporal bones. The first draft of the paper was written by B.B. and B.W. All authors contributed to writing portions of the manuscript and approving the final submitted version.

\section{Data Availability Statement}

The datasets generated during and/or analyzed during the current study are available in the figures but can be obtained also from the corresponding author upon reasonable request.

\section{References}

Akbal OY, Kaymaz C, Tanboga IH, Hakgor A, Yilmaz F, Turkday S, et al. Extrinsic compression of left main coronary artery by aneurysmal pulmonary artery in severe pulmonary hypertension: its correlates, clinical impact, and management strategies. Eur Heart J Cardiovasc Imaging. 2018;19:1302-8.

Aw ST, Fetter M, Cremer PD, Karlberg M, Halmagyi GM. Individual semicircular canal function in superior and inferior vestibular neuritis. Neurology. 2001;57:768-74.

Baloh RW. Clinical practice. Vestibular neuritis. N Engl J Med. 2003;348:1027-32.

Baloh RW, Kerber KA. Clinical neurophysiology of the vestibular system. 4th ed. New York: Oxford University Press; 2011.

Buki B, Ward BK. Length of the narrow bony channels may not be the sole cause of differential involvement of the nerves in vestibular neuritis. Otol Neurotol. 2021;42:e918-e924.

Duker JS, Brown GC. Anterior location of the crossing artery in branch retinal vein obstruction. Arch Ophthalmol. 1989;107:998-1000.

Fedorov A, Beichel R, Kalpathy-Cramer J, Finet J, Fillion-Robin JC, Pujol S, et al. 3D slicer as an image computing platform for the quantitative imaging network. Magn Reson Imaging. 2012;30:1323-41.

Fonseca AC, Albuquerque L, Ferro JM. Reversible bilateral sensorineural hearing loss in a woman with cerebral venous thrombosis. J Neurol. 2008;255:1825-6.

Gattringer T, Enzinger C, Birner A, Wünsch G, Niederkorn K, Walch C, et al. Acute unilateral hearing loss as an early symptom of lateral cerebral sinus venous thrombosis. Arch Neurol. 2012;69:1508-11.
Gianoli G, Goebel J, Mowry S, Poomipannit P. Anatomic differences in the lateral vestibular nerve channels and their implications in vestibular neuritis. Otol Neurotol. 2005;26:48994.

Goddard JC, Fayad JN. Vestibular neuritis. Otolaryngol Clin North Am. 2011;44:361-viii.

Goebel JA, O’Mara W, Gianoli G. Anatomic considerations in vestibular neuritis. Otol Neurotol. 2001;22:512-8.

Guo R, Zhang H, Chen W, Zhu X, Liu W, RaskAndersen $\mathrm{H}$. The inferior cochlear vein: surgical aspects in cochlear implantation. Eur Arch Otorhinolaryngol. 2016;273:355-61.

Hayreh SS. Pathogenesis of optic disc edema in raised intracranial pressure. Prog Retin Eye Res. 2016;50:108-44.

Horton KM, Talamini MA, Fishman EK. Median arcuate ligament syndrome: evaluation with CT angiography. Radiographics. 2005;25: 1177-82.

Hussain MA, Aljabri B, Al-Omran M. Vascular thoracic outlet syndrome. Semin Thorac Cardiovasc Surg. 2016;28:151-7.

Iyer JS, Zhu N, Gasilov S, Ladak HM, Agrawal SK, Stankovic KM. Visualizing the 3D cytoarchitecture of the human cochlea in an intact temporal bone using synchrotron radiation phase contrast imaging. Biomed Opt Express. 2018; 9:3757-67.

Jeong SH, Kim HJ, Kim JS. Vestibular neuritis. Semin Neurol. 2013;33:185-94.

Joussen AM. Retinal vascular disease. Berlin; NY: Springer; 2007.

Kim HA, Sohn SI, Lee H. Cerebral venous thrombosis mimicking acute unilateral vestibulopathy. Neurol Sci. 2008;29:41-3.
Kim SJ, Reed N, Betz JF, Abraham A, Lee MJ, Sharrett AR, et al. Association between microvascular retinal signs and age-related hearing loss in the atherosclerosis risk in communities neurocognitive study (ARIC-NCS). JAMA Otolaryngol Head Neck Surg. 2020;146:152-9.

Kimura R, Perlman HB. Extensive venous obstruction of the labyrinth. B. Vestibular changes. Ann Otol Rhinol Laryngol. 1956;65: 620-38.

Kimura R, Perlman HB. Extensive venous obstruction of the labyrinth. A cochlear changes. Ann Otol Rhinol Laryngol. 1956;65:332-50.

Kimura RS, Trehey JA, Hutta J. Vein of the vestibular aqueduct in the gerbil. Acta Otolaryngol. 1996;116:44-51.

Kleffner I, Dörr J, Ringelstein M, Gross CC, Böckenfeld Y, Schwindt W, et al. Diagnostic criteria for susac syndrome. J Neurol Neurosurg Psychiatry. 2016;87:1287-95.

Mazzoni A. Vein of the vestibular aqueduct. Ann Otol Rhinol Laryngol. 1979;88:759-67.

Mazzoni A. The vascular anatomy of the vestibular labyrinth in man. Acta Otolaryngol Suppl. 1990;472:1-83.

Mei X, Atturo F, Wadin K, Larsson S, Agrawal S, Ladak HM, et al. Human inner ear blood supply revisited: the Uppsala collection of temporal bone-an international resource of education and collaboration. Ups J Med Sci. 2018; 123:131-42.

Mizisin AP, Kalichman MW, Myers RR, Powell HC. Role of the blood-nerve barrier in experimental nerve edema. Toxicol Pathol. 1990; 18:170-85.

Nabeya D. A study in the comparative anatomy of the blood-vascular system of the internal ear in mammalia and in homo. Sch Med Univ Imp Kyoto. 1923;6:1-132. 
Nadol JB Jr. Vestibular neuritis. Otolaryngol Head Neck Surg. 1995;112(1):162-72.

Pogson JM, Taylor RL, Young AS, McGarvie LA, Flanagan S, Halmagyi GM, et al. Vertigo with sudden hearing loss: audio-vestibular characteristics. J Neurol. 2016;263:2086-96.

Rambold H, Boenki J, Stritzke G, Wisst F, Neppert B, Helmchen C. Differential vestibular dysfunction in sudden unilateral hearing loss. Neurology. 2005;64:148-51.

Rujescu D, Hartmann AM, Giegling I, Konte B, Herrling M, Himmelein S, et al. Genomewide association study in vestibular neuritis: involvement of the host factor for HSV-1 replication. Front Neurol. 2018;9:591.

Schuknecht HF. Pathology of the ear. Philadelphia: Lea \& Febiger; 1993.

Schuknecht HF, Merchant SN, Nadol JB. Schuknecht's pathology of the ear. 3rd ed. Shelton, CT: People's Medical Pub. HouseUSA; 2010.
Scott IU, Campochiaro PA, Newman NJ, Biousse V. Retinal vascular occlusions. Lancet. 2020; 396(10266):1927-40.

Sheyman A, Fawzi AA. Retinal vascular disease: Springer Singapore; 2020. p. 156.

Shrier I, Magder S. Pressure-flow relationships in in vitro model of compartment syndrome. J Appl Physiol. 1995;79:214-21.

Song T, Zhang D. Evaluation on curative effects of ethylene diamine tetra-acetic acid chelation therapy in treating with atherosclerotic cardiovascular disease: a protocol for systematic review and meta-analysis. Medicine. 2020;99(52):e23346.

Staurenghi G, Lonati C, Aschero M, Orzalesi N. Arteriovenous crossing as a risk factor in branch retinal vein occlusion. Am J Ophthalmol. 1994;117:211-3.
Strum D, Kapoor E, Shim T, Kim S, Sabetrasekh P, Monfared A. Prevalence of sensorineural hearing loss in pediatric patients with sickle cell disease: a meta-analysis. Laryngoscope. 2021;131(5):1147-56.

Tanaka T, Shimizu K: Retinal arteriovenous shunts in Takayasu disease. Ophthalmology. 1987;94:1380-8.

Tarnutzer AA, Bockisch CJ, Buffone E, Weber KP. Association of posterior semicircular canal hypofunction on video-head-impulse testing with other vestibulo-cochlear deficits. Clin Neurophysiol. 2017;128:1532-41.

Taylor RL, McGarvie LA, Reid N, Young AS, Halmagyi GM, Welgampola MS. Vestibular neuritis affects both superior and inferior vestibular nerves. Neurology. 2016;87:1704-12.

Weiss P. Endoneurial edema in constricted nerve. Anat. Rec.. 1943;86(4):491-522.

Yanagihara N, Honda N, Hato N, Murakami S. Edematous swelling of the facial nerve in Bell's palsy. Acta Otolaryngol. 2000;120:66771. 